\title{
Components for an Integrated Recirculating Frequency Shifter Device (NNX16AD14G-Y3)
}

\author{
SHAYAN MOOKHERJEA \\ Department of Electrical and Computer Engineering, University of California, San Diego, MC 0407 La \\ Jolla CA 92093-0407 USA \\ Email: smookher@ucsd.edu
}

We report and discuss measurements of silicon photonics components which comprise a recirculating variable-count frequency shifter (RVCFS) device. Summary of a Project Outcomes report of research funded by NASA.

\section{Introduction and Goals}

The objective of this project is to develop the technology for silicon photonic transceiver components that can comprise a coherent laser optical communication and/or relay network in near-Earth space at wavelengths near $1550 \mathrm{~nm}$, for use on a satellite platform. Our proposed transceiver architecture generates a flexible number of communication streams from a single laser, which can be demultiplexed and individually modulated at the transmitter and decoded at the receiver. This scalability supports both high bit-rate communications with orders-of-magnitude reductions in size, weight, and energy consumption. Our strategy offers improvements on link performance and bandwidth compared to both RF communications and existing optical technologies.

\section{Activities}

Last year, we showed a breadboard demonstration of the recirculating variablecount frequency shifter (RVCFS) which can generate a variable number of tones (ranging from 1 to about 10) [1]. The breadboard experiment helps validate the RVCFS concept, and provides a benchmark for the microchip version.

The recirculating variable-count frequency shifter (RVCFS) comprises of a single-sideband carrier-suppressed (SSBCS) modulator driven by an externally-supplied RF tone, a tunable optical bandpass filter, and a gain section i.e., optical amplifier, which are placed in a loop.

The primary research activity this year was the design of an integrated silicon microchip which contains several of the components (excluding the gain block) in a single photonic device. A layout of the chip is shown in Fig. 1 and a photograph of the chip is shown in Fig. 2. The GDS uses $32 \mathrm{~mm}^{\wedge} 2$ fabrication area out of the $50 \mathrm{~mm}^{\wedge} 2$ total wafer fabrication space within the scope of the original research proposal 


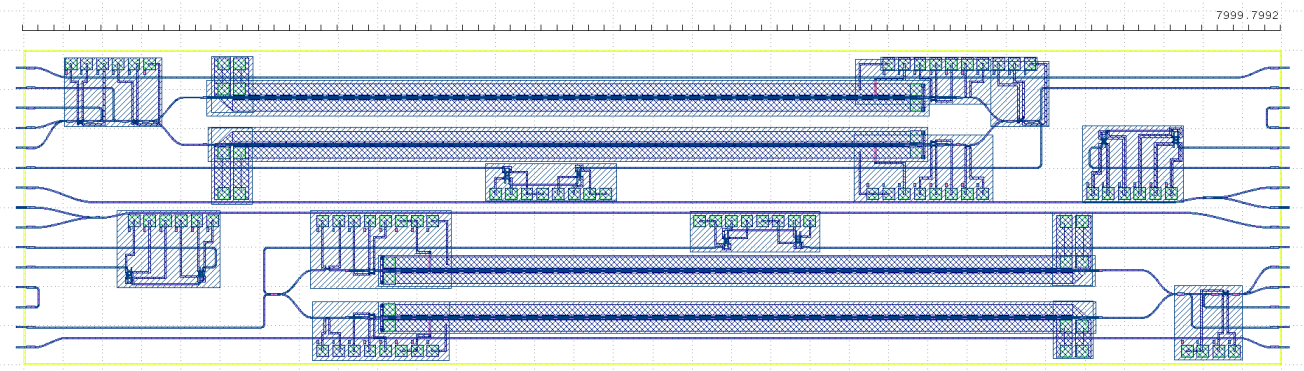

Figure 1 Layout of the silicon photonics implementation of the RVCFS component excluding the gain block. Edge couplers are used to couple to fibers along the east and west edges of the chip. Two copies of the design are placed within the design area.

Unfortunately, there was a flaw in the layout, which consisted of a gap between two unconnected waveguide segments near a bend. This was not caught by a manual pre-fabrication design check, and no automated design check was performed on the GDS. Consequently, there was a very high additional propagation loss (about $20 \mathrm{~dB}$ ) on top of the approximately 10-15 dB fiber to fiber loss that is typical of chips fabricated using Sandia's silicon photonics process. This is too much gain to make up using amplification. We diced out the separate components (modulator and filters) that comprise the optical circuit and tested them separately (with additional insertion loss since the edge couplers now lack tapers).

The filter response is shown in Fig. 3 and shows a reasonably high extinction ratio (adequate for the recirculating loop application) and a low insertion loss; thus, they performed satisfactorily. However, the phase shifters (which are part of the single-sideband electro-optic modulators) work at a lower bandwidth $(<$ $8 \mathrm{GHz})$ than ideal $(\sim 20 \mathrm{GHz})$. An alternative design which we developed in a different project, which integrates thin-film lithium niobate with silicon photonic passive circuits, showed better performance and an ultrahigh modulation bandwidth that is beyond the capabilities of traditional depletionmode Mach-Zehnder modulators[2].

The "control knob" over the number of tones is the center frequency of the optical loop filter, not the number of laser diodes fabricated on the chip. Previously, we have studied optical wave propagation and bandpass filtering using the silicon coupled-resonator optical waveguide structure[3], [4], which uses relatively low quality-factor resonators coupled in a serial cascade. However, such higher-order filtering devices have a complex dispersion relationship[5]-[7] and are inherently sensitive to fabrication disorder. In earlier stages of this project, we simulated the expected performance degradation from using non-ideal (i.e., not brick wall) filters (see Year 1 report). These insights were further developed using the breadboard experiment (see Year 2 report). 


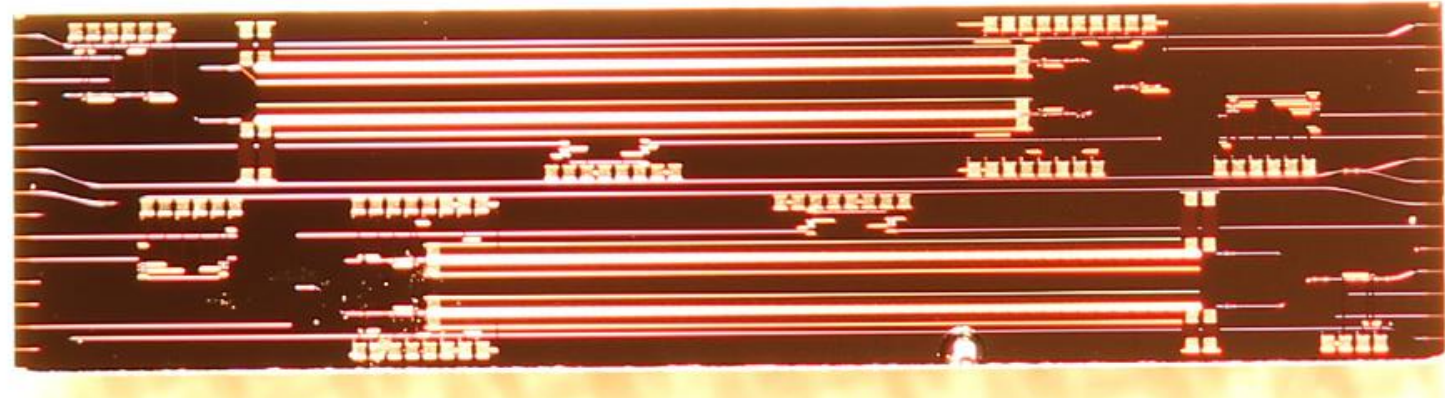

Figure 2 Camera image of a received photonic chip ( $8 \mathrm{~mm} \times 2 \mathrm{~mm} ; 2$ similar design variants are included in this section), for the integrated RFS structure (with external optical gain). The chip uses edge couplers with spot-size converters to match to polarization maintaining highnumerical-aperture fiber arrays.

\section{Impact}

Our research shows an innovative concept in wavelength-division multiplexing (WDM). Compared to multiple lasers, the advantage of this approach to generating multiple tones are many-fold. We do not require multiple lasers but are able to implement WDM using a single laser. We enable more efficient power usage. We achieve granular (line by line) control over the number of carriers, which can enable user-defined dynamic communications rate adjustment over a very wide range.

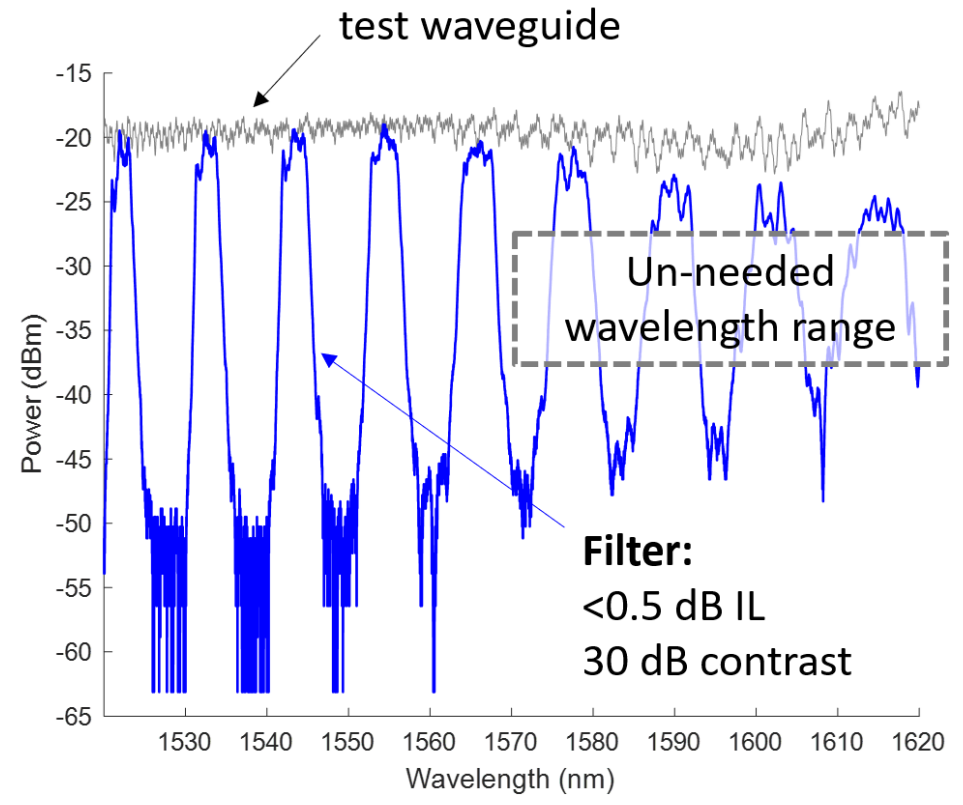

Figure 3 Transmission of an optical filter section (fifth order coupled microring filter) that is part of the microchip. This segment was diced out and tested separately.

This scheme also has an important fundamental advantage: in a communication system, the bit rate is reduced typically when the signal-to-noise ratio is low. 
Under those circumstances, channel capacity benefits from boosting the signal level (e.g., Shannon's channel capacity formula). This happens automatically in this scheme: when the filter bandwidth is narrow (i.e., fewer number of carriers, and lower overall bit rate), all the amplifier gain is dedicated to boosting the few circulating tones in the loop. This may help address the nearfar communications problem in space, where large variations in the distance between source and destination should be expected.

The components in the loop should have uniform performance across the bandwidth that spans all the tones, which could range from a few nanometers to several nanometers, depending on the RF frequency used to shift the tones in each pass through the SSB modulator. There are several couplers in the architecture which must be carefully designed, fabricated, and potentially trimmed after fabrication in the integrated chip.

Since the single-sideband generation process results in tone-generation only to one side (here, the shorter frequency side) of the original carrier, we can achieve the same results as narrowing the filter bandwidth by moving the center frequency of the filter over towards higher frequencies (and not worrying about the filter passband width). This has the effect of compressing the available bandwidth for comb generation between the filter roll-off edge on the left and the carrier tone on the right. This itself results in a variable loop bandwidth, and this scheme is substantially easier (and lower insertion loss) than the technique of varying the optical filter bandwidth by programmable optical filters.

The work of one graduate student at UCSD was supported by this project. A self-funded masters student also contributed to research. The students received training in the fields of electrical engineering, physics, device fabrication and measurement. Results have been published in peer-reviewed journals and presented at internationally-recognized conferences, as well as workshops, seminars, and meetings.

\section{Potential Subsequent Extensions}

One of the challenges of high index contrast integrated photonics is the sensitivity to disorder. This is particularly true for the coupled-resonator configuration[6], [7] and affects the extinction ratio of filters. However, we have also studied ways to study and potentially compensate for disorder after fabrication [8]-[10]. We tested both a second-order filter structure, with two sections whose center wavelength can be thermally tuned independently from each other using integrated metal heaters, and a fifth order filter structure. The 2nd order microring filter has about $4 \mathrm{~dB}$ insertion loss when not tuned (i.e., the rings are at different center frequencies); this is expected to be $<1 \mathrm{~dB}$ insertion loss when the chips are wirebonded and the FPGA tune-up algorithm is implemented. The typical extinction ratio was seen to be about 7-10 $\mathrm{dB}$. The 
5th order filters have a better response even without tuning (as shown in Fig. 3) with insertion loss $<0.5 \mathrm{~dB}$ and extinction $>25 \mathrm{~dB}$.

Figure 4 shows a camera image of test chips mounted to a custom-designed printed circuit board (PCB), with RF probes approaching to test the electrooptic modulation properties. DC connections are wire bonded from the chip to the $\mathrm{PCB}$, and a header connection is provided on the right-hand side edge of the PCB.

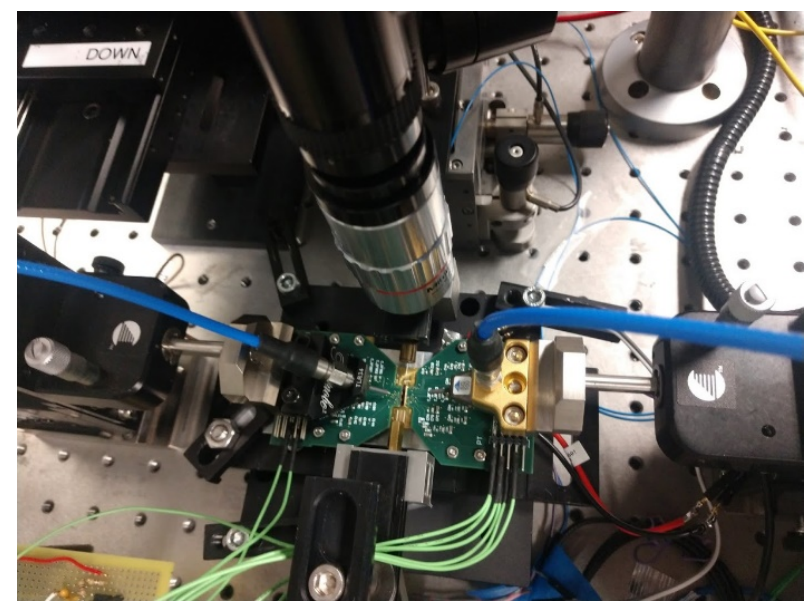

Figure 4 Selected silicon photonic microchips were incorporated onto high-speed multi-layer PCBs with required driver electronics, connectors and headers. The setup shows the testing of chips underway using laboratory instrumentation.

Figure 5 shows the design of the PCB, which included decoupling capacitors and routing of electrical lines. Delivery and control of electrical power to the chip requires off-board buffer ICs, DACs/ADCs, and interfaces to a microcontroller.

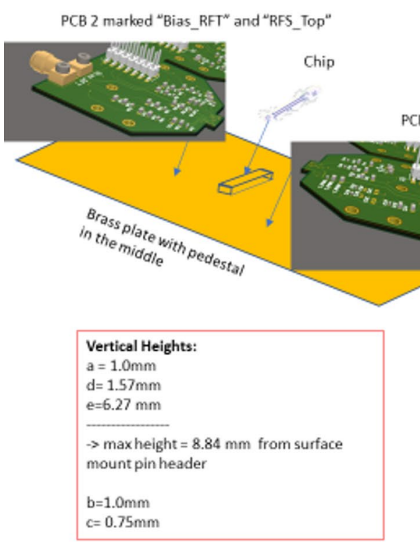

PCB 1 marked "Bias_RFB" and "RFS Bottom"

whel

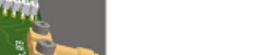


Using the diced-out segments of the standard dopant profile test chips, we were able to see clear evidence of modulation at $10 \mathrm{GHz}$ (the target for the ESI project), but the performance was better at lower frequencies e.g., $5 \mathrm{GHz}$ and we estimated a 3-dB electrical roll-off somewhere around 7-8 GHz. We also observed good single sideband extinction of $>30 \mathrm{~dB}$, which is currently better than the state of the art silicon photonic single sideband modulator. We also measured chips from a second batch, which have a different dopant profile, and these have 3-dB bandwidth beyond $10 \mathrm{GHz} 3-\mathrm{dB}$. As shown in Fig. 6, the sideband amplitude is about $+4 \mathrm{~dB}$ higher in this case, which translates into increased efficiency of the RFS concept. The trade-off is a slightly higher insertion loss incurred by optical carrier in propagating through the higherdoped regions.

Physics theory suggests that there are other interesting Kerr nonlinear effects that might occur in appropriately dispersion-engineered devices, such as ways to prevent the spreading of light through soliton generation [11], [12]. However, these effects are mostly beyond the scope of this project. We have also not addressed how the optical gain block can be integrated on the same chip as the modulator and filter. Since the SSB modulator is polarization sensitive, it would be helpful to integrate the amplifier on the same chip so that the state of polarization does not drift or become misaligned.

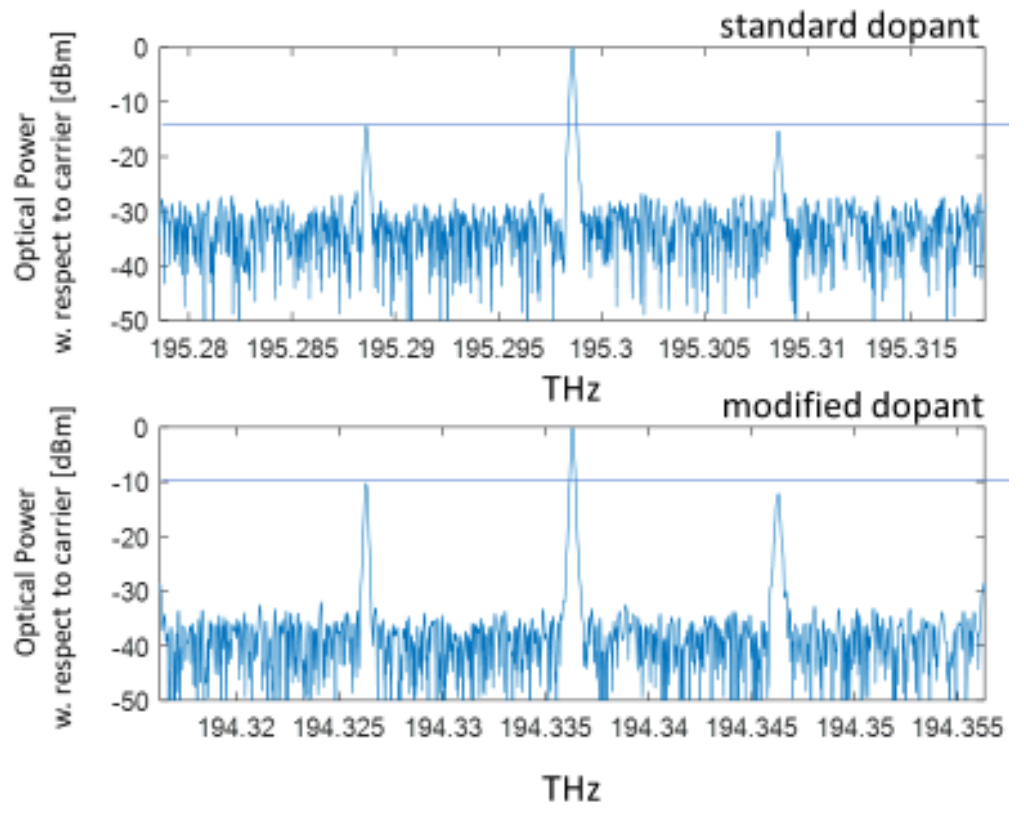

Figure 6 Test of electro-optic Mach-Zehnder modulators at $10 \mathrm{GHz}$, using two different p-n junction dopant concentrations used on our silicon photonic chip variants. Compared to the standard process, the higher-concentration $(2 \mathrm{x})$ dopant process yields about $4 \mathrm{~dB}$ improvement, in terms of conversion from carrier to sideband but also has higher optical loss, which adversely impacts the loop performance. 
Figure 7 shows the gradual tuning of the single-sideband modulator from an initial balanced state (when only one of the Mach-Zehnder modulator structures was driven) into the optimal operating point with both MZM's driven with the correct phase relationship, at which $30 \mathrm{~dB}$ sideband suppression was observed.

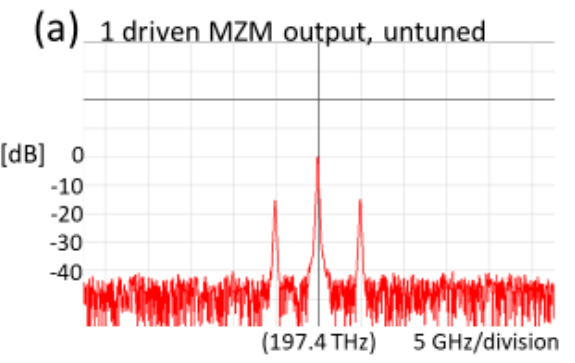

(b) 2 driven MZMs output, untuned

(c) Tuning to suppress sideband and carrier, optimizing sideband
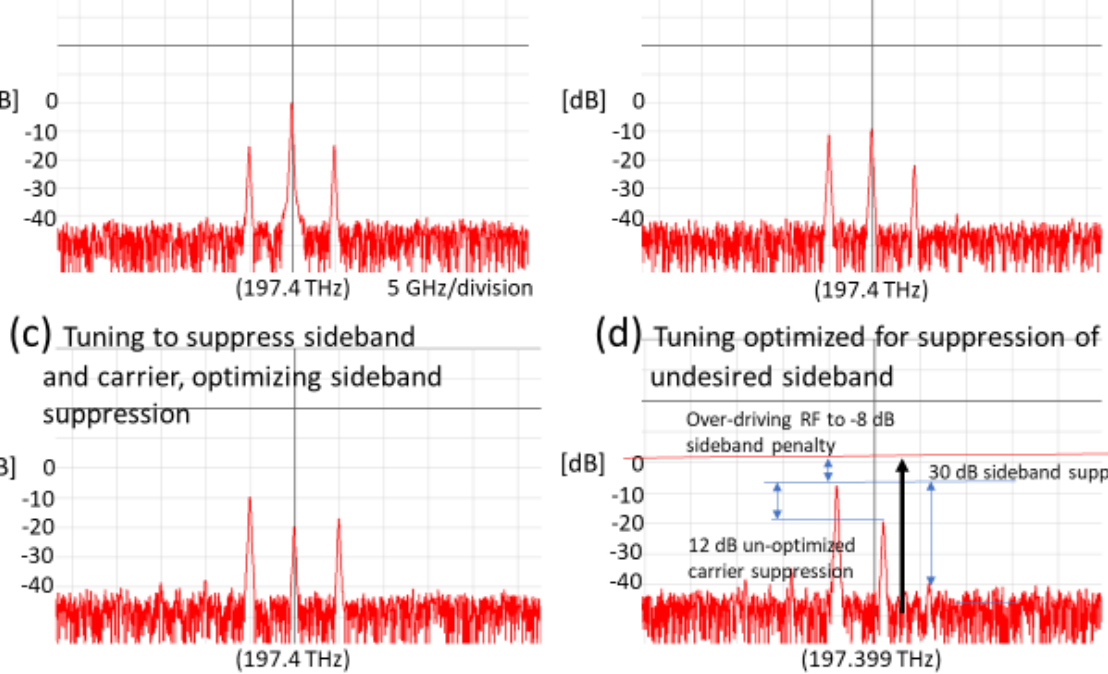

(d) Tuning optimized for suppression of undesired sideband

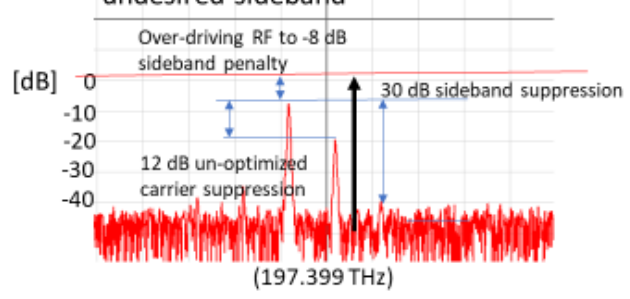

Figure 7 Sequence of phase and bias tuning for single sideband electro-optic modulation.

\section{Open-Access Reporting Initiative}

PRAISE: This open-access document is provided in support of our PRAISE

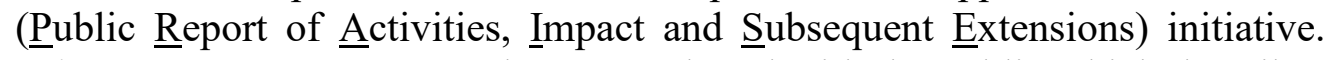
What is it? An open-access document shared with the public which describes the research outcomes of publicly-funded projects such as those funded by the U.S. NSF (National Science Foundation) and other agencies.

\section{References}

[1] X. Wang and S. Mookherjea, "Performance comparisons between semiconductor and fiber amplifier gain assistance in a recirculating frequency shifter," Opt. Lett., vol. 43, no. 5, p. 1011, Mar. 2018, doi: 10.1364/OL.43.001011.

[2] X. Wang, P. O. Weigel, J. Zhao, M. Ruesing, and S. Mookherjea, "Achieving beyond-100-GHz large-signal modulation bandwidth in hybrid silicon photonics Mach Zehnder modulators using thin film lithium niobate," APL Photonics, vol. 4, no. 9, p. 096101, Sep. 2019, doi: 10.1063/1.5115243. 
[3] M. L. Cooper et al., "235-ring Coupled-Resonator Optical Waveguides," in Conference on Lasers and Electro-Optics 2010, San Jose, California, 2010, p. CTuHH3. doi: 10.1364/CLEO.2010.CTuHH3.

[4] J. R. Ong and S. Mookherjea, "Quantum light generation on a silicon chip using waveguides and resonators," Opt. Express, vol. 21, no. 4, p. 5171, Feb. 2013, doi: 10.1364/OE.21.005171.

[5] S. Mookherjea, "Using gain to tune the dispersion relation of coupledresonator optical waveguides," IEEE Photon. Technol. Lett., vol. 18, no. 5, pp. 715-717, Mar. 2006, doi: 10.1109/LPT.2006.871144.

[6] S. Mookherjea and M. A. Schneider, "Avoiding bandwidth collapse in long chains of coupled optical microresonators," Opt. Lett., vol. 36, no. 23, p. 4557, Dec. 2011, doi: 10.1364/OL.36.004557.

[7] M. L. Cooper and S. Mookherjea, "Modeling of Multiband Transmission in Long Silicon Coupled-Resonator Optical Waveguides," IEEE Photon. Technol. Lett., vol. 23, no. 13, pp. 872-874, Jul. 2011, doi: 10.1109/LPT.2011.2141657.

[8] M. L. Cooper, G. Gupta, J. S. Park, M. A. Schneider, I. B. Divliansky, and S. Mookherjea, "Quantitative infrared imaging of silicon-on-insulator microring resonators," Opt. Lett., vol. 35, no. 5, p. 784, Mar. 2010, doi: 10.1364/OL.35.000784.

[9] S. Mookherjea and H. R. Grant, "High dynamic range microscope infrared imaging of silicon nanophotonic devices," Opt. Lett., vol. 37, no. 22, p. 4705, Nov. 2012, doi: 10.1364/OL.37.004705.

[10] Y. Shen, I. B. Divliansky, D. N. Basov, and S. Mookherjea, "Perfect set-and-forget alignment of silicon photonic resonators and interferometers," in Optical Fiber Communication Conference/National Fiber Optic Engineers Conference 2011, Los Angeles, California, 2011, p. PDPC3. doi: 10.1364/OFC.2011.PDPC3.

[11] B. Crosignani, A. Yariv, and S. Mookherjea, "Nonparaxial spatial solitons and propagation-invariant pattern solutions in optical Kerr media," Opt. Lett., vol. 29, no. 11, p. 1254, Jun. 2004, doi: 10.1364/OL.29.001254.

[12] S. Trillo and W. Torruellas, Spatial solitons. 2001. Accessed: Jan. 02, 2022. [Online]. Available:

http://public.ebookcentral.proquest.com/choice/publicfullrecord.aspx? $\mathrm{p}=3$ 088063 